\title{
New evidence on the nature of the encoding of action events
}

\author{
LARS BÄCKMAN, LARS-GÖRAN NILSSON, and DAVID CHALOM \\ University of Umea, Umea, Sweden
}

\begin{abstract}
Differences in recall patterns between subject-performed tasks (SPTs) and verbal materials have been interpreted in terms of SPTs being nonstrategic or automatically encoded. In a series of three experiments, we tested this notion by comparing free recall of SPTs and sentences in conditions of (1) nondivided versus divided attention for organizable items, (2) organizable versus nonorganizable items, and (3) nondivided versus divided attention for nonorganizable items. It was found that recall of both SPTs and sentences decreased in conditions of divided attention. A decrease in recall was also observed for both types of material when nonorganizable as compared to organizable materials were used. In addition, the degree of clustering was higher for SPTs than for sentences. These data suggest that there is a strategic component involved in the encoding of SPTs. We propose that the action elements of SPTs (e.g., motor features, shape, texture) are automatically encoded, whereas the verbal component is strategically encoded. It is emphasized that organization is an encoding strategy critical to SPT recall.
\end{abstract}

An experimental task for memory research was introduced by Cohen in 1981. In this task, labeled subjectperformed task (SPT), subjects are instructed to perform a series of miniature actions for a subsequent memory test. SPTs involve either (1) one or two objects provided by the experimenter (e.g., "bounce the ball," "sharpen the pencil") or (2) no objects at all (e.g., "clap your hands," "snap your fingers"). Using this task, Cohen (1981, 1983), Cohen and Bean (1983), and Cohen and Stewart (1982) observed several deviations from established memory laws that are valid for verbal materials.

First, Cohen (1981) demonstrated that the normally obtained effect of manipulations regarding levels of processing on recall of words (e.g., Craik \& Tulving, 1975) did not apply to recall of SPTs. A second difference between verbal recall and SPT recall is that the reliable primacy effect associated with free recall of verbal materials has not appeared in SPT recall (Cohen, 1981; Cohen \& Bean, 1983; Cohen \& Stewart, 1982). Cohen (1981) explained this phenomenon as being due to the presence of active memorization strategies (rehearsal) leading to a primacy effect for memory of words, and an absence of these strategies in encoding of SPTs, resulting in an absence of primacy. A third difference relates to the developmental effects typically found in recall of verbal materials. Whereas significant differences are usually obtained in recall, for example, of words as a function of increased

This research was supported by grants from the Bank of Sweden Tercentenary Foundation and the Swedish Council for Research in the Humanities and the Social Sciences. The authors wish to thank James J. Jenkins, Roberta L. Klatzky, Richard S. Newman, and two anonymous reviewers for providing constructive comments on an earlier version of this article.

Reprint requests should be addressed to Lars Bäckman, Department of Psychology, University of Ume̊̊, Rådhusesplanaden 2, S-902 47 Umeá, Sweden. age, Cohen and Stewart (1982) found this untrue for SPT recall among 9-, 11-, and 13-year-old children.

On the basis of these differences between verbal recall and SPT recall, Cohen (1981) suggested that the encoding mechanisms in "verbal memory models" are of little importance in SPT recall. This was supported by the fact that although subjects in the Cohen (1981) study reported active attempts to memorize the words (usually by means of rehearsal), no such attempts were reported in the case of SPTs.

Moreover, on the basis of Brown's (1975) claim that strategic tasks should show developmental differences, Cohen and Stewart (1982) interpreted the absence of developmental effects in SPT recall as supportive of the nonstrategic nature of SPTs. This interpretation was supported by the Cohen and Bean (1983) study in which educable mentally retarded subjects were compared to nonretarded controls: a significant deficit in immediate word recall, but not in immediate SPT recall, was shown by the retarded subjects. Since retarded subjects were presumed to have general strategic deficits and consequently were expected to prove disadvantaged in strategic memory tests, these results were taken as a further indication that SPT encoding is nonstrategic.

This hypothesis was specifically tested in two experiments (Cohen, 1983). In the first experiment, the subjects' attention during the learning phase of a free recall task was manipulated so that more attention was focused on some items, designated as being especially important, than on others. This manipulation had a greater effect on word recall than on SPT recall. In the second experiment, subjects' accuracy in ability to predict recall of SPTs, as compared to recall of verbal materials, was tested. Subjects' ratings showed good predictive power for word recall in subsequent tests, but not for SPT recall. Cohen suggested from these data "that words and SPTs represent 
two anchor positions on a continuum of memory events... whose dimensions may be strategy use (as suggested by the serial position data) and/or automaticity in encoding (as suggested by the results of both experiments)" (Cohen, 1983, p. 581, italics added).

In this context, Cohen (1983) referred to a similar continuum proposed by Hasher and Zacks (1979) that progresses from genetically "prepared" automatic processes (requiring no attention), through automatic processes developed by means of practice, to effortful operations (demanding attention), thereby suggesting that use of strategies (as with verbal materials) is highly effortful, whereas absence of the use of strategies (as in the case of SPTs) points toward automaticity of encoding (Cohen, 1983).

However, on the basis of a series of recent studies from our own laboratory (Bäckman, 1985; Bäckman \& Nilsson, 1984, 1985), we have arrived at a somewhat different conclusion regarding the nature of the encoding of SPTs. A task analysis of SPTs in relation to verbal memory tasks reveals differences on two fundamental dimensions. First, SPTs are multimodal in the sense that several sensory systems are involved during encoding. The experimenter reads each SPT aloud, thereby presenting the information auditorily. The visual system is involved throughout the presentation/performance of SPTs. Subjects are instructed to act motorically and, accordingly, the tactual mode is activated. Also, some SPTs (e.g., "smell the perfume," "eat the raisin") bring about activity in the olfactory and gustatory modes. Second, each SPT comprises a variety of features on which encoding may be based. In addition to verbal features, there are features of color, shape, texture, and sound; also, there are motor features, since subjects are instructed to act motorically. It should be noted that some of these features (e.g., shape, motor features) are nominally present in two modalities, whereas others (e.g., color, texture) are modality-specific. Clearly, the multimodal and contextually rich properties of SPTs distinguish SPTs from standard verbal memory tasks, in which the presentation typically is unimodal and the number of features is limited to semantic, phonemic, and graphic aspects of the materials. Accordingly, there are at least two kinds of information that can be stored in the SPT task: the verbal commands and the motor actions. In the case of verbal memory tasks, only the former type of information is available.

Although we agree with Cohen (e.g., 1981, 1983) that the action component of SPTs is likely to be automatically encoded (cf. Kausler, 1983; Kausler \& Hakami, 1983), we argue that the verbal component of SPTs, similar to that of verbal memory tasks, is subject to strategic encoding operations. There are two kinds of evidence in favor of this assumption. First, Bäckman and Nilsson $(1984,1985)$ measured the degree of organization of SPTs and verbally presented sentences in terms of SPTs/sentences involving objects or not. The major finding in both of these studies was that SPTs were organized to a much greater extent than were sentences. We interpreted the superior organization of SPTs as being due to the multimodal and contextually rich properties of this memory task. That is, these properties are supposed to enhance the possibility of detecting and utilizing the superordinate categories on which organization may be based (Bäckman \& Nilsson, 1984, 1985).

Second, it is well known in the memory literature (see Murdock, 1974, for a review) that the asymptote of the serial-position curve is sensitive to various organizational variables, such that easily organizable to-be-remembered (TBR) materials increase the level of the asymptote. An inspection of Cohen's data (Cohen, 1981; Cohen \& Stewart, 1982) and our own data (Bäckman \& Nilsson, $1984,1985)$ reveals that the level of the asymptote is generally higher for SPTs than for various verbal control tasks, causing the leveling out of the whole prerecency section of the serial-position curves for SPTs. That is to say, the absence of primacy relative to the asymptote does not necessarily imply an absence of use of strategies at encoding of SPTs. Taken together, organizational data and serial-position data suggest that Cohen's conceptualization of SPT encoding may have been premature.

To obtain further knowledge on this issue, we conducted a series of three experiments. In all experiments, free recall of SPTs and sentences (depicting the same verbal information) was tested. In Experiment 1, subjects learned categorically organizable SPTs and sentences under conditions of nondivided and divided attention. In Experiment 2, organizable and nonorganizable (not associable by categories) SPTs and sentences were used. Finally, in Experiment 3, subjects learned nonorganizable SPTs and sentences under conditions of nondivided and divided attention.

The variables manipulated in these experiments are in line with the criteria used to evaluate effortful and automatic processes in Hasher and Zacks's (1979) framework. As mentioned, Cohen (1983) relates his own continuum to that of Hasher and Zacks (1979), under the assumption that automatically encoded events correspond to nonstrategic events, whereas effortfully encoded events correspond to strategic events. According to Hasher and Zacks, one cardinal feature of automatic processes is that they should allow other effortful processes to occur simultaneously with minimal disruption. Effortful processes, on the other hand, are attention-demanding and supposed to be limited in efficiency in divided-attention tasks.

To study potential differences in organization of SPTs and sentences, we used a list of items that could be organized according to five semantic categories in Experiment 1 . The use of semantic categories stems from Bäckman and Nilsson's $(1984,1985)$ notion that SPTs, compared to verbal memory tasks, enable a superior organization of items, for example, according to SPTs performed with or without objects (as demonstrated in the two cited studies). These studies, however, were not designed specifically for the purpose of studying organization. In Experiment 1 in the present study, organization of SPTs and sentences was examined on an a priori 
basis using five semantic categories with five subordinates each. Experiment 2 was designed so that the organizational variable was explicitly manipulated. Specifically, the purpose of Experiment 2 was to investigate the effect that withdrawal of the possibility to organize according to semantic category would have on SPT and sentence recall. The purpose of Experiment 3 was to study the effect of divided/nondivided attention using materials that were not easily organizable. The hypothesis was that the detrimental effects of divided attention would be more pronounced for nonorganizable items.

Taking Cohen's $(1981,1983)$ view that SPTs are nonstrategic and verbal tasks are strategic as a point of departure, one would not expect SPTs to be affected by manipulations of nondivided/divided attention, whereas one would expect sentences to be so affected. Moreover, one would not expect organizable, as opposed to nonorganizable items, to lead to higher recall performance in the SPT task, whereas one would expect this in the sentence task.

On the other hand, on the basis of our contention that SPT encoding comprises a strategic component, we expect that both SPT recall and sentence recall will be impaired under conditions of divided attention. In addition, given that organization is a strategy more likely to be successfully utilized at encoding of SPTs than of sentences, we expect that (1) SPTs will be more impaired than sentences when the TBR items are not easily organizable, and (2) the degree of clustering will be higher for SPTs than for sentences.

\section{EXPERIMENT 1}

\section{Method}

Design. The design was a $2 \times 2$ (type of task $\times$ type of material) factorial. Types of task were (1) nondivided attention (NDA) and (2) divided attention (DA). Types of material were (1) SPTs and (2) bimodally presented sentences (Ss). The experiment thus comprised four conditions: NDA/SPTs, NDA/Ss, DA/SPTs, and DA/Ss.

Subjects. Eighty students from the University of Umeả served as subjects, with random assignment of 20 subjects to each of the four conditions. Age ranges were: 19-36 (Mean = 24.4) for NDA/SPTs, 18-35 (Mean = 23.8) for NDA/Ss, 17-41 (Mean = 24.1) for DA/SPTs, and 19-31 (Mean $=22.5$ ) for DA/Ss. All subjects were paid the equivalent of $\$ 4$ (U.S.) for participating in the experiment, which lasted approximately $25 \mathrm{~min}$.

Materials. A list of 25 sentences in the imperative form was used. The list consisted of five different categories of imperatives, with five subordinates in each, referring to actions involving (1) parts of the body (e.g., "clap your hands," "bite your lip"), (2) articles of clothing (e.g., "put on the glove," " "put the boot on the floor"), (3) pen and paper (e.g., "write a number," "draw a triangle"), (4) toys (e.g., "shake the rattle," "bounce the ball"), and (5) kitchen utensils (e.g., "lift the cup," "put the cover on the pot"'). All items were counterbalanced in the list, so that one item from each category was included in each of five serial-position blocks. The order of items was randomized within blocks. Two presentation orders were used, each administered to half the subjects in each condition.

Procedure. The list described above constituted the basis for the two different types of material. Accordingly, in two conditions, the TBR items consisted of the list of bimodally presented sentences, whereas in the other two conditions, subjects were asked to per- form the SPTs. In the bimodal events, the sentences were presented consecutively on a screen by means of a slide projector, at a rate of $5 \mathrm{sec}$ each and with an interstimulus interval (ISI) of $1 \mathrm{sec}$. The items were also read aloud simultaneously by the experimenter. For the SPT events, the subjects sat at a table screened from a second table on which the objects used in the minitasks were placed. The experimenter presented each SPT by instructing the subject what to do; when an object was required for the performance of an SPT, this was presented together with the task instruction. For example, the experimenter presented a ball with the instruction "bounce the ball." Rate of presentation and ISIs were the same as for sentences ( $5 \mathrm{sec}$ and $1 \mathrm{sec}$, respectively). The experimenter kept the rate and the ISI constant with the help of prerecorded signals played on a tape recorder.

Subjects assigned to the NDA conditions were told either (1) that a list of 25 sentences would be presented on a screen and simultaneously read aloud by the experimenter, or (2) that they would be asked to perform 25 short and simple tasks. They were also told to remember as many SPTs/sentences as possible for a free recall test to be taken immediately after the last item on the list. No mention was made of the fact that the items belonged to five semantic categories.

Subjects assigned to the two DA conditions received the same instructions. In addition, they were informed of a secondary task: in addition to remembering the items, they were told to count backward aloud during the performance/presentation of the items. The counting task consisted of subtracting 6 and 3 , in turn, from a number $(600)$ given by the experimenter $7 \mathrm{sec}$ before the first item appeared. The subjects were told that they had two assignments to fulfill: (1) to memorize the items and (2) to reach as low a number as possible in their backward counting. They were also instructed that under no circumstances whatsoever were they to interrupt the backward counting during the experimental session. A trial count from the number $300(294,291,285,282$, etc. $)$ was requested to ensure the subjects' understanding of the instructions.

After the presentation of the last item, all subjects received an immediate free recall test in which any recall order was permitted. A total of $10 \mathrm{~min}$ was allowed for this test. After the experimental session, all subjects were questioned about their use of memory strategies (e.g., organization, rehearsal by means of repeating the items, imagery). This interview was maintained in all three experiments reported in this article

\section{Results and Discussion}

A lenient scoring procedure for measuring SPT and sentence recall was adopted in all three experiments. That is, responses were accepted if their meaning was correct (cf. Cohen, 1981; Cohen \& Stewart, 1982). Recency items (21-25) were not scored, so that scoring was based only on the first 20 items of the recall lists; this was done because the main purpose of the experimental series was to examine potential differences between SPTs and sentences in the degree to which strategies were employed. By not scoring recency items, the influence of short-term memory was minimized. These scoring criteria were retained in all three experiments.

The data obtained in Experiment 1 are portrayed in Figure 1. As can be seen in this figure, SPT recall is higher than sentence recall, and performance for both tasks decreases when attention is divided during encoding.

A general significance level of $p<.05$ was adopted for all statistical analyses in the experiments to be reported. The recall data of Experiment 1 were analyzed with a $2 \times 2 \times 2$ (task [NDA, DA] $\times$ material [SPTs, sen- 


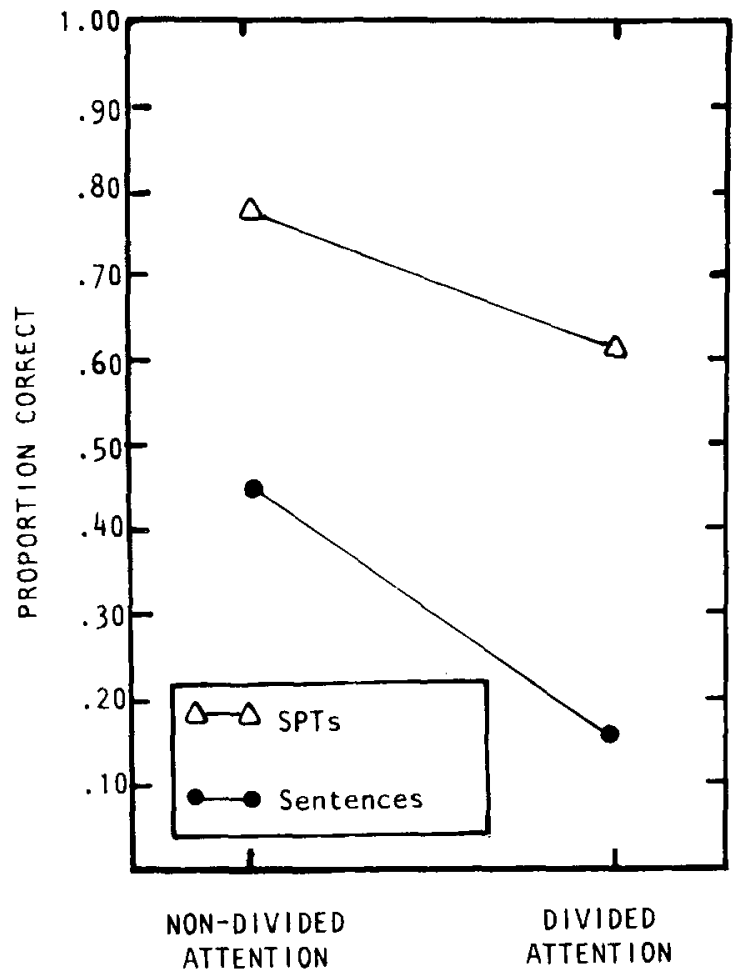

Figure 1. Mean proportion correct free recall of organizable SPTs and sentences in conditions of nondivided and divided attention (Experiment 1).

tences] $\times$ order of presentation) analysis of variance (ANOVA). All factors varied between subjects. No effects of order were obtained, and hence, the data were collapsed over this variable. The ANOVA revealed significant main effects of task $[F(1,72)=107.02$, $M S \mathrm{e}=3.83]$ and material $[F(1,72)=311.92$, $M S e=3.83$ ]. In addition, the material $\times$ task interaction was reliable $[F(1,72)=5.49, M S e=3.83]$.

An a posteriori Tukey test revealed that subjects recalled significantly more SPTs and sentences under conditions of NDA compared to DA. An inspection of Figure 1 indicates that the interaction between task and material is due to a greater decrease of sentence recall than of SPT recall in the DA condition.

In order to study potential differences between recall of SPTs and sentences in degree of categorical organization, an adjusted ratio of clustering (ARC) score analysis (Roenker, Thompson, \& Brown, 1971) was conducted. Level of clustering in each condition is presented in Table 1. It appears that SPTs are organized to a greater extent than are sentences, and furthermore, that there is a disproportionate decrease in organization of sentences in the DA condition.

A $2 \times 2$ ANOVA, with the factors referring to task and

Table 1

Mean Adjusted Ratio of Clustering (ARC) Scores for SPTs and Sentences in Conditions of Nondivided and Divided Attention

\begin{tabular}{ccc}
\multicolumn{3}{c}{ Sentences in Conditions of Nondivided and Divided Attention } \\
\hline & SPTs & Sentences \\
\hline Nondivided Attention & 0.48 & 0.33 \\
Divided Attention & 0.46 & -0.10 \\
\hline
\end{tabular}

material, was conducted on the ARC score data. A significant main effect of material was found: SPTs were organized to a higher degree than were sentences $[F(1,76)$ $=8.44, \mathrm{MSe}=0.30$ (mean ARC scores $=47.0$ and 11.5 for SPTs and sentences, respectively)]. However, the main effect of task and the task $\times$ material interaction were not reliable.

The data of the present experiment clearly show an effect of the divided attention manipulation: recall performance for both SPTs and sentences decreased considerably in the DA condition. According to Hasher and Zacks (1979), an effortful task, such as the backward counting used here, should interfere with other effortful operations but not with automatic processes. Thus, the fact that both SPT and sentence recall were impaired under DA conditions suggests that there are strategic components involved in the encoding of SPTs as well as of sentences.

Two other aspects of these data are notable: the general superiority of SPT recall compared to verbal recall, and the fact that sentence recall decreased more than SPT recall under DA conditions.

We hypothesized that there are two kinds of information that can be represented in the case of SPTs: (1) the: verbal commands and (2) the multimodal and contextually rich motor actions involving features of color, texture, shape, and sound. Whereas the former type of information is supposed to be strategically encoded, the latter type of information is supposed to be acquired automatically (cf. Kausler, 1983; Kausler \& Hakami, 1983). We attribute the superior recall of SPTs to the multimodal and rich properties of this task. Conceivably, these properties bring about a richer representation of the TBR information and, ultimately, a higher level of recall, as compared to the sentence task, in which only verbal information is available.

Furthermore, the multimodal and contextually rich properties of SPTs are supposed to enhance the possibility of detecting and utilizing the semantic categories on which organization is based. It could be the case that SPT recall decreased to a lesser extent than sentence recall in the DA condition, because the high degree of "organizability" of SPTs counteracted the detrimental effects of DA.

\section{EXPERIMENT 2}

The TBR information used in Experiment 1 was composed of imperatives from five different semantic categories. As discussed, that composition of lists was selected so that the subjects would be able to demonstrate the use of organizational strategies. In addition to organizable TBR items, another set of items was used in Experiment 2 . This set was composed of 25 imperatives, each from a different semantic category; these iterns could not easily be organized into clusters as in Experiment 1.

Given that organization is an encoding strategy more likely to be successfully employed for SPTs than for sentences, one would expect the reduction of recall for nonorganizable as opposed to organizable items to be more 
pronounced for SPTs than for sentences. According to Cohen's (e.g., 1981, 1983) contention that SPTs are nonstrategically encoded, whereas verbal materials are strategically encoded (by means of, for example, organization), exactly the opposite interaction between task and material would be expected.

\section{Method}

Design. The experiment employed a $2 \times 2$ (type of task $\times$ type of material) factorial design. Type of task was either organizable (O) or nonorganizable (NO) items, while SPTs and sentences were used as material. This experiment thus comprised four conditions: O/SPTs, O/Ss, NO/SPTs, and NO/Ss.

Subjects. Eighty students from the University of Umeå were randomly assigned to the four conditions, 20 subjects to each. Age ranges were: $18-39($ Mean $=24.1)$ in O/SPTs, $19-30($ Mean $=$ 22.5 ) in $\mathrm{O} / \mathrm{Ss}, 18-33$ (Mean $=22.6$ ) in NO/SPTs, and 17-43 (Mean $=24.5$ ) in NO/Ss. All subjects were paid the equivalent of $\$ 4$ (U.S.) for participation. The experiment lasted approximately $25 \mathrm{~min}$.

Materials. The same list of items used in Experiment 1 was again used in the two organizable conditions. For the two nonorganizable conditions, a new list of items was prepared consisting of 25 different sentences in the imperative form. These items belonged to different semantic categories. Subjects assigned to the NO/Ss condition were presented with these sentences bimodally (visually and auditorily), whereas subjects assigned to the NO/SPTs condition were requested to perform the actions implied by the sentences. Rate of presentation and ISIs were $5 \mathrm{sec}$ and $1 \mathrm{sec}$, respectively, for all groups. Two presentation orders were used, with half the subjects in each condition assigned to each order.

Procedure. For both SPT conditions, the experiment was carried out in the same manner as the NDA/SPTs condition in Experiment 1 . For both conditions using sentences, the procedure was the same as in the NDA/Ss condition in Experiment 1.

\section{Results and Discussion}

Figure 2 presents the data obtained in the recall test. It can be seen in this figure that the use of a NO list is associated with a decrease in memory performance, which is more pronounced for SPTs than for sentences.

The ANOVA carried out on these data was a $2 \times 2 \times 2$ (task $\times$ material $\times$ order) factorial. No order effects were obtained; thus, the data were collapsed over this variable. The ANOVA revealed main effects of task $[F(1,72)=$ $30.63, M S e=4.50]$ and material $[F(1,72)=121.37$, $M S e=4.50]$. The interaction between task and material was also reliable $[F(1,72)=4.67, M S e=4.50]$.

An a posteriori Tukey test revealed that both SPTs and sentences were recalled significantly better when using organizable than when using nonorganizable items. An inspection of Figure 2 indicates that the interaction between task and material is caused by greater impairment of SPTs than of sentences in the NO condition.

On the basis of this interaction, we conclude that Cohen's (1983) contention that SPTs are nonstrategically encoded is not supported. SPT recall was impaired more than were sentences by removal of the option to organize, suggesting that SPT recall is largely dependent on organization. Since the use of organization as a strategy is an effortful process (e.g., Hasher \& Zacks, 1979), it may

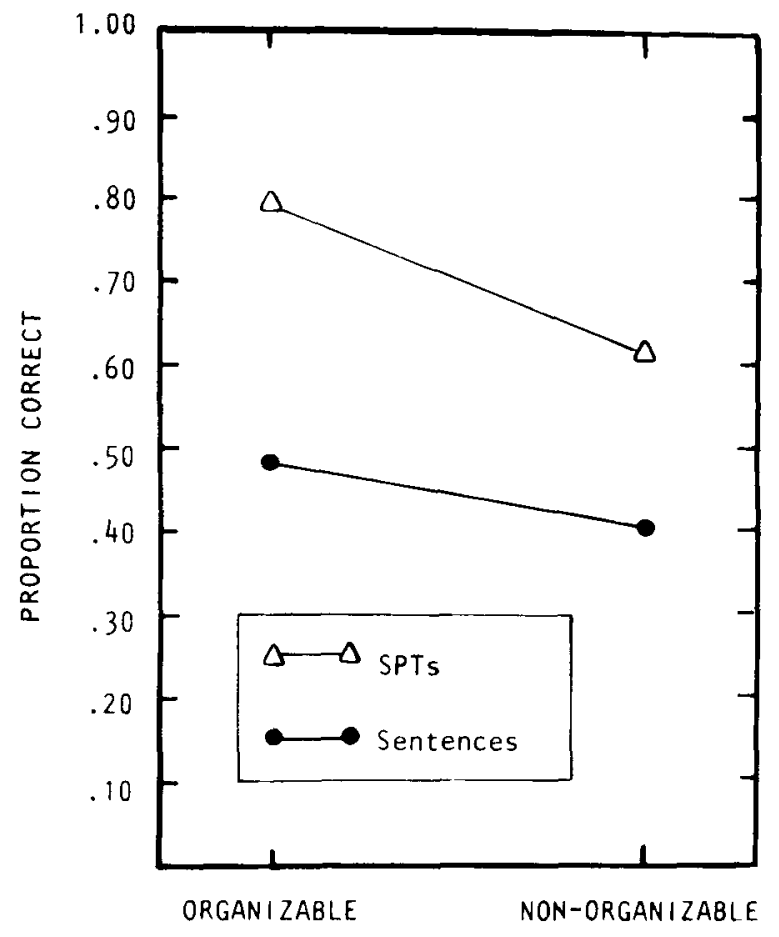

Figure 2. Mean proportion correct free recall of SPTs and sentences for organizable and nonorganizable materials (Experiment 2).

be concluded that there is a strategic component involved in the encoding of SPTs. In other words, the present findings are in agreement with the contention that one of the main factors involved in the encoding of SPTs is organization. This organization is supposed to be enhanced by the multimodal and rich properties of SPTs, enabling discovery and successful utilization of the categories on which organization is based.

As can be seen in Figure 2, the removal of the option to organize the TBR information is not as detrimental for sentence recall as it is for SPT recall. There are two possible explanations for this result. First, it may be that subjects assigned to the $\mathrm{O} / \mathrm{Ss}$ condition did, indeed, try to organize the sentences. However, this strategy may be implemented less successfully with sentences than with SPTs because the multimodal and rich properties that aid organizational processing are lacking. Second, some strategy other than organization may have been used at encoding of sentences, hence compensating for the lack of organizability. One such strategy could be rehearsal by means of repetition of items. At the conclusion of the experiment, subjects were interviewed about their use of strategies. In these interviews, $40 \%$ of the subjects in the two sentence conditions reported that they tried to memorize the items by repeating them, but no such attempts were reported in the two SPT conditions (see Cohen, 1981, for similar results).

Obviously, these two explanations of the relatively smaller decrease in sentence recall than in SPT recall when the material is nonorganizable are not mutually exclusive. Instead, it is arguable that the less "organizable nature" 
of sentences as well as the use of other encoding strategies contribute to the interaction between SPTs/sentences and organizable/nonorganizable materials.

In two experiments, we have demonstrated support for the contention that strategic components are involved in the encoding of SPTs. However, not all data are unequivocal. In the DA condition in Experiment 1, a smaller decrease in SPT recall compared with sentence recall was obtained. This was not expected, and the question is why such an effect was obtained. As mentioned, the multimodal and rich properties of SPTs may enhance the possibility for successful organization to occur. As a consequence, the potential of SPTs to be organized may, to some extent, have compensated for the detrimental effects of divided attention on recall.

\section{EXPERIMENT 3}

In order to determine the validity of the above reasoning, Experiment 3 was conducted. The conditions were identical to those of Experiment 1, with the exception that the materials used were nonorganizable, so as to remove the option of organizing the items. Recall of nonorganizable SPTs and sentences was tested in conditions of nondivided and divided attention.

We reasoned that an outcome showing the same magnitude of impairment of SPT recall and sentence recall when attention is divided would be in line with the above hypothesis.

\section{Method}

Design. A $2 \times 2$ (type of task $\times$ type of material) factorial design was employed. All factors were the same as in Experiment 1; that is, type of task was either NDA or DA, and type of material was either SPTs or sentences. This experiment thus comprised four conditions as well: NDA/SPTs, NDA/Ss, DA/SPTs, and DA/Ss.

Subjects. Fifty-six students from the University of Umea were randomly assigned to the four conditions (14 each). Age range for each group was: NDA/SPTs $=18-27($ Mean $=21.1), \mathrm{NDA} / \mathrm{Ss}$ $=18-28($ Mean $=23.6)$, DA/SPTs $=17-35($ Mean $=23.0)$, and $\mathrm{DA} / \mathrm{Ss}=17-29($ Mean $=22.4)$. These subjects were also paid the equivalent of $\$ 4$ (U.S.) for their participation. The experiment lasted approximately $25 \mathrm{~min}$.

Materials. The list of items that was used in Experiment 2 in the conditions with nonorganizable items was used in this experiment. There were two presentation orders.

Procedure. The same procedure used in Experiment 1 was used in this experiment.

\section{Results and Discussion}

The data of Experiment 3 are summarized in Figure 3. It can be seen in this figure that (1) there is a superior recall performance for SPTs in both task conditions, and (2) the two functions depicted are parallel; that is, the difference between SPT recall and sentence recall is equivalent for both levels of the task variable.

The $2 \times 2 \times 2$ (task $\times$ material $\times$ order) ANOVA revealed no order effects, and the data were collapsed over this variable. There were significant main effects of task

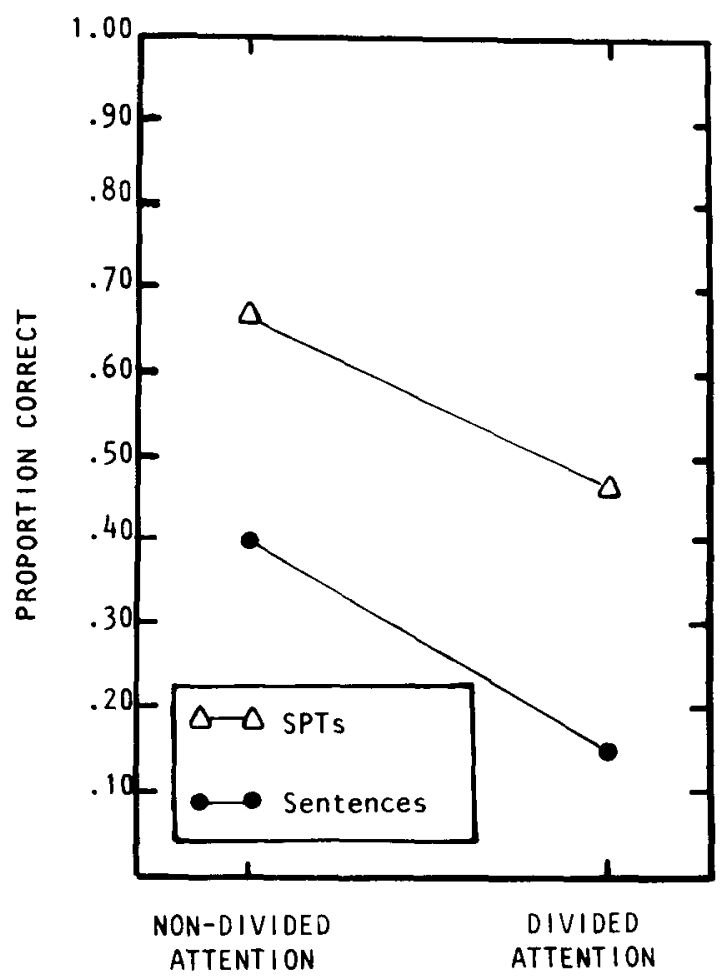

Figure 3. Mean proportion correct free recall of nonorganizable SPTs and sentences in conditions of nondivided and divided attention (Experiment 3).

$[F(1,48)=144.86, M S e=2.08]$ and material $[F(1,48)$ $=236.19, M S \mathrm{e}=2.08]$. Most importantly, the task $\times$ material interaction fell far short of significance $(p>.20)$.

In this experiment, the TBR information was composed of items that could not easily be associated with each other into clusters as in Experiment 1, where the materials were composed of groups of related imperatives. Thus, the only procedural difference between Experiments 1 and 3 is the composition of the TBR information used; the difference in results between these two experiments is that a task $x$ material interaction was obtained in Experiment 1, but not in Experiment 3. Comparing recall performance for SPTs and sentences in the critical DA conditions (see Figures 1 and 3), it can be seen that the performance level for sentences is about the same in the two experiments, but not for SPTs. When the TBR materials are composed of imperatives that cannot easily be organized into clusters (Experiment 3), the performance is about $15 \%$ lower than when a list of organizable items is used (Experiment 1). This comparison suggests to us that the highly organizable nature of SPTs prevented the detrimental effects of divided attention on recall in Experiment 1. Finally, provided that impaired recall in dual-task performance is a characteristic feature of strategic memory tasks (Hasher \& Zacks, 1979), Experiments 1 and 3 provide strong evidence against the notion that encoding of SPTs is an entirely nonstrategic operation. 


\section{GENERAL DISCUSSION}

The three experiments reported in this article have demonstrated that (1) recall of SPTs is generally superior to recall of bimodally presented sentences comprising the same verbal information as the SPTs; (2) sentence recall decreases more than SPT recall under conditions of divided, compared with nondivided, attention for organizable items, although a significant decrease was observed for both types of material; (3) the level of clustering is higher for SPTs than for sentences; (4) SPT recall decreases more than sentence recall when the items are nonorganizable, compared with organizable, although a significant decrease was observed for both types of material; and (5) SPT recall and sentence recall are equally impaired under conditions of divided attention for nonorganizable items.

These findings suggest that there is a strategic component involved in the encoding of SPTs, and that SPTs as well as sentences fall on the "effortful side" of the Hasher and Zacks (1979) continuum. Thus, the present data do not support Cohen's (e.g., 1981, 1983) claim that SPTs are nonstrategically encoded. Rather, the results call for a more differentiated conception of the nature of the encoding of action events.

The position taken here is that the action component of SPTs (including color, shape, motor features, texture, and sounds) is encoded nonstrategically, whereas the verbal component (i.e., the imperatives) is encoded strategically. Furthermore, we argue that organization is an encoding strategy more likely to be successfully employed at encoding of SPTs than of sentences, the reason being that the nonverbal features of SPTs (e.g., motor features, color, shape) enhance the possibility to utilize organizational strategies. In other words, due to the multimodal and contextually rich properties of SPTs, "organizational schemata" are established, promoting deliberate organization of the verbal commands.

Analogously, the lack of a multimodal and rich encoding environment in the case of sentences makes the organizational processing more difficult to carry out. That is, it could be that subjects try to engage in organizational processing to comparable extents in sentences and SPTs, but that this strategy is implemented more successfully in the case of SPTs. Furthermore, perhaps other encoding strategies, such as rehearsal by means of repetition, are more likely to be used for sentences than for SPTs. The presentation of verbal items in the visual or auditory modality affords that subjects repeat the items in order to improve memory; that is, rehearsal may be a strategy that is more compatible with the task demands of sentences than of SPTs. As noted, there are interview data supporting this suggestion (see also Cohen, 1981).

This conception of the encoding of action events and the proposed differences between the encoding of action events and verbal events fit the results of the present experiments quite well. This is illustrated by the following four main findings of the present research. First, the generally superior recall of SPTs is attributed to the multimodal and rich properties of this task (action components and verbal component). These properties supposedly bring about a richer representation of the TBR items, which in turn leads to a higher level of recall as compared with sentences where only the verbal component is available for encoding.

Second, the highly organizable nature of SPTs explains why SPTs decreased to a lesser extent than did sentences under conditions of divided attention for organizable items; that is, the organizability prevented, to some extent, the negative effects of divided attention on recall. In the same vein, the superior clustering of SPTs is attributed to the multimodal and rich nature of SPT inputs that would seem to enhance successful organization.

Third, given that organization plays a more important role in the encoding of SPTs than in verbal encoding, it follows that the recall differences between SPTs and sentences decrease when the option to organize the materials is withdrawn. This was exactly what happened in Experiment 2 .

Finally, when the high organizability of SPTs cannot compensate for the negative effects of divided attention (Experiment 3), our theory predicts that the strategic encoding of the verbal components of SPTs and sentences will suffer to the same degree, as was the case in Experiment 3 . Although it is clear that the encoding operations carried out in Experiment 3 are attention-demanding and effortful (see Figure 3), it should be pointed out that the qualitative nature of these operations is not yet known. Future research may illuminate this issue.

Viewed from another theoretical perspective, it could be argued that SPTs and verbal materials differ in the extent to which the strategies used are initiated or provoked by the environmental support at hand, or stem from selfinitiated constructive operations (Craik, 1983). We claim that (1) SPTs and verbal memory tasks differ in the sense that SPTs are multimodal and contextually rich, whereas verbal memory tasks typically are unimodal and contextually poorer, and (2) the multimodal and rich features of SPTs more readily permit the use of organizational strategies; thus, we submit that SPTs and verbal memory tasks differ in their location on a continuum ranging from maximal provision of environmental support to maximal need of self-initiated operations (see Craik, 1983). That is, the organizational strategies employed when remembering SPTs are strongly guided by the specific properties of each task, whereas the strategies employed for remembering verbal events are dependent to a greater extent on subjects' self-initiated activities.

An alternative interpretation of the present data is that the organization of SPTs takes place, exclusively, at retrieval. Such a notion would be in agreement with the Kausler (1983) model of rehearsal-dependent and rehearsal-independent memory, which states that action events (e.g., SPTs) are similar to verbal events in that both are subject to strategic retrieval operations (see also Cohen, 1984; Cohen \& Bean, 1983). We argue, however, 
that a "pure" retrieval explanation of the present data is less plausible, and the reason for this is twofold. First, subjects in all three experiments were questioned about their use of strategies at both encoding and retrieval. These data are not extensively reported in this article. For present purposes, the most interesting aspect of these interviews is that concerned with the reported use of organizational strategies at encoding and/or retrieval for subjects assigned to SPTs. There are three conditions that are irrelevant in this respect (condition of nonorganizable items in Experiment 2, and both conditions in Experiment 3), the reason being that the materials used here were composed so as to minimize the possibility for organization. In the remaining three conditions, $90 \%$ of the subjects reported use of organization. Of these, $25 \%$ reported that they organized the SPTs only at encoding, $35 \%$ reported organization only at retrieval, whereas $40 \%$ reported organization at both phases.

Although these data should be treated with due caution, it is obvious that they support the notion that organization is a strategy likely to be employed for SPTs. Also, it seems reasonable to argue that the subjective reports are at odds with the claim that SPTs are organized only at retrieval, since $58 \%$ of the subjects in these conditions reported that they grouped the SPTs into semantic categories during the learning phase.

Moreover, there is a second argument against the notion that only SPT retrieval is a strategic operation: namely, the fact that SPT recall decreased significantly under conditions of divided attention (Experiments 1 and 3). Obviously, subjects' attention was divided only at encoding, and it seems to us that the most parsimonious explanation of the decrease of recall is that the backward counting interfered with the deliberate encoding of the verbal commands of SPTs (cf. Hasher \& Zacks, 1979).

In conclusion, the present experimental series suggests that there are strategic memory operations involved in the encoding of SPTs. Specifically, it is argued that the verbal component of the SPT task is subject to strategic operations, whereas the action component of SPTs is encoded in the absence of encoding strategies.

\section{REFERENCES}

BäcKMAN, L. (1985). Further evidence for the lack of adult age differences on free recall of subject performed tasks: The importance of motor action. Human Learning, 4, 79-87.

BäCKMAN, L., \& NiLsson, L.-G. (1984). Aging effects in free recall: An exception to the rule. Human Learning, 3, 53-69.

Bäckman, L., \& Nilsson, L.-G. (1985). Prerequisites for lack of age differences in memory performance. Experimental Aging Research, 11, 67-73.

Brown, A. L. (1975). The development of memory: Knowing, knowing about knowing, and knowing how to know. In H. W. Reese (Ed.), Advances in child development and behavior (Vol. 10, pp. 103-152). New York: Academic Press.

CoHEN, R. L. (1981). On the generality of some memory laws. Scandinavian Journal of Psychology, 22, 267-282.

CoHEN, R. L. (1983). The effect of encoding variables on the free recall of words and action events. Memory \& Cognition, 11, 573-582.

COHEN, R. L. (1984). Individual differences in event memory: A case for nonstrategic factors. Memory \& Cognition, 12, 633-641.

Cohen, R. L., \& Bean, G. (1983). Memory in educable mentally retarded adults: Deficit in subject or experimenter? Intelligence, 7 , 287-298.

COHEN, R. L., \& STEwart, M. (1982). How to avoid developmental effects in free recall. Scandinavian Journal of Psychology, 23, 9-16.

CraIK, F. I. M. (1983). On the transfer of information from temporary to permanent memory. Philosophical Transactions of the Royal Society of London, 302, 341-359.

Craik, F. I. M., \& Tulving, E. (1975). Depth of processing and the retention of words in episodic memory. Joumal of Experimental $P_{s y-}$ chology: General, 104, 268-294.

HASHER, L. Z ZACKs, R. T. (1979). Automatic and effortful processes in memory. Journal of Experimental Psychology: General, 108, 356-388.

KAUSLER, D. H. (1983, August). Episodic memory and human aging. Invited address, American Psychological Association Meetings, Anaheim, CA.

KAUSLER, D. H., \& HAKAMI, M. K. (1983). Memory for activities: Adult age differences and intentionality. Developmental Psychology, 19, 889-894.

MURDOCK, B. B., JR. (1974). Human memory: Theory and data. Potomac, MD: Erlbaum.

Roenker, D. L., Thompson, C. P., \& Brown, S. C. (1971). Comparisons of measures for the estimation of clustering in free recall. Psychological Bulletin, 76, 45-48.

(Manuscript received June 21, 1985; revision accepted for publication December 30, 1985.) 